\title{
World Brain Day 2020: Join Us to "Move to End Parkinson's Disease": A World Federation of Neurology and International Parkinson and Movement Disorders Society Collaboration
}

\author{
Tissa Wijeratne (D), Susan Fox
}

Keywords: World Brain Day, Parkinson disease, Brain health, Advocacy

doi: $10.1017 / \operatorname{cjn} .2020 .140$

Can J Neurol Sci. 2021; 48: 56-58

All of us are enveloped in the current health and humanitarian emergency due to the COVID-19 pandemic. Despite the crisis, it is critical for us to find our way through and bring out the best of humanity as physicians. It is in this setting that we have written this commentary on brain health and World Brain Day (WBD) 2020.

Neurological disorders are the leading cause of disability and second leading cause of mortality worldwide. ${ }^{1}$ Despite these alarming statistics, the gaps, and disparities in the distribution of neurological resources and therapeutics worldwide are enormous. The global median of the total neurological workforce defined as the number of neurologists, neurosurgeons, and child neurologists available in a country is only 3.1 per 100,000 inhabitants. Low-income countries report a median of 0.1 per 100,000 inhabitants, compared with 7.1 per 100,000 in highincome countries.

One of the best ways to address these disparities is through widespread global advocacy and campaigns. The WBD is an annual activity of the World Federation of Neurology (WFN) held on July 22 commemorating the establishment of the WFN in 1957. This is a large global event for awareness and advocacy of brain health and neurosciences. The previous topics for WBD were "Our brains our future" in 2014, "epilepsy" (partnering with International League Against Epilepsy in 2015, "Brain Health and the Ageing Population" in 2016, "Stroke is a brain attack - prevent it and treat it" (partnering with World Stroke Organisation (WSO) in 2017, "Clean air for brain health" in 2018 (partnering with the WFN Environmental Neurology Specialty Group), and "Migraine, the painful truth" (partnering with International Headache Society (IHS) in 2019. (For details, please see the WFN website (https://wfneurology.org/ world-brain-day-2020). This year the focus of WBD is Parkinson's disease (PD).

$\mathrm{PD}$ is the fastest growing brain disorder affecting the world. ${ }^{2}$ The Global Burden of Diseases (GBD) study noted that 6.2 million people currently have PD. The number of people with PD more than doubled from 1990 to 2015 . $^{2}$ In Canada, PD affects 1 in every 500 people. $^{3}$ The current GBD estimates of PD in
Canada are shown, with the kind permission of Institute for Health Metrics and Evaluation (Figures 1 and 2).

$\mathrm{PD}$ is a complex movement disorder resulting in motor and non-motor symptoms that significantly impact the everyday lives of patients and their caregivers. A Canadian study conducted by Wang and colleagues noted that the majority of people with PD reported a negative impact on interaction with others, suffered economic impact, and required assistance from caregivers. ${ }^{3}$ Currently, due to the COVID-19 pandemic, social distancing, lockdowns, medication supply issues, and reduced access to health care have made life generally harder for PD patients and caregivers. However, the pandemic has created some new opportunities. Increased virtual consults and novel methods of accessing multidisciplinary care are becoming more commonly available as a result of the innovation these times have inspired.

How can we respond to the hidden pandemic of PD that seems to be doubling at an alarming rate? History seems to show us the way forward. Just as the community changed behaviour to stop the spread of COVID-19, focused effort on resources and knowledge gaps in research for PD need to be addressed as a matter of priority. Thus, as the health care systems re-arranged infrastructure, teams, systems of health care delivery in the middle of the pandemic (with no proven cure within our sight), the access to optimal care for PD worldwide must be offered as a matter of urgency. Just as the funders and policy-makers devoted funding towards a potential vaccine against COVID-19, potential therapeutics, and diagnostics

From the School of Psychology and Public Health, La Trobe University, Melbourne, Australia (TW); Department of Neurology, Western Health \& University Melbourne, AIMSS, Level three, WHCRE, Sunshine Hospital, St Albans, Australia (TW); Department of Medicine, Faculty of Medicine, University of Rajarata, Saliyapura, Anuradhapura, Sri Lanka (TW); Neurology, University Health Network and Sinai Health System, Toronto ON, Canada (SF); Neurology, Krembil Research Institute, UHN, Toronto ON, Canada (SF); Neurology, University of Toronto, Toronto ON, Canada (SF); and Movement Disorder Clinic, Edmond J. Safra Program in Parkinson Disease, Toronto Western Hospital, Toronto, ON, Canada (SF)

Received June 12, 2020. Final Revisions Submitted July 1, 2020. Date of Acceptance July 3, 2020.

Correspondence to: Tissa Wijeratne, Department of Neurology, Western Health \& University Melbourne, AIMSS, Level three, WHCRE, Sunshine Hospital, St Albans, 3021, Australia. Email: tissa.wijeratne@wh.org.au 


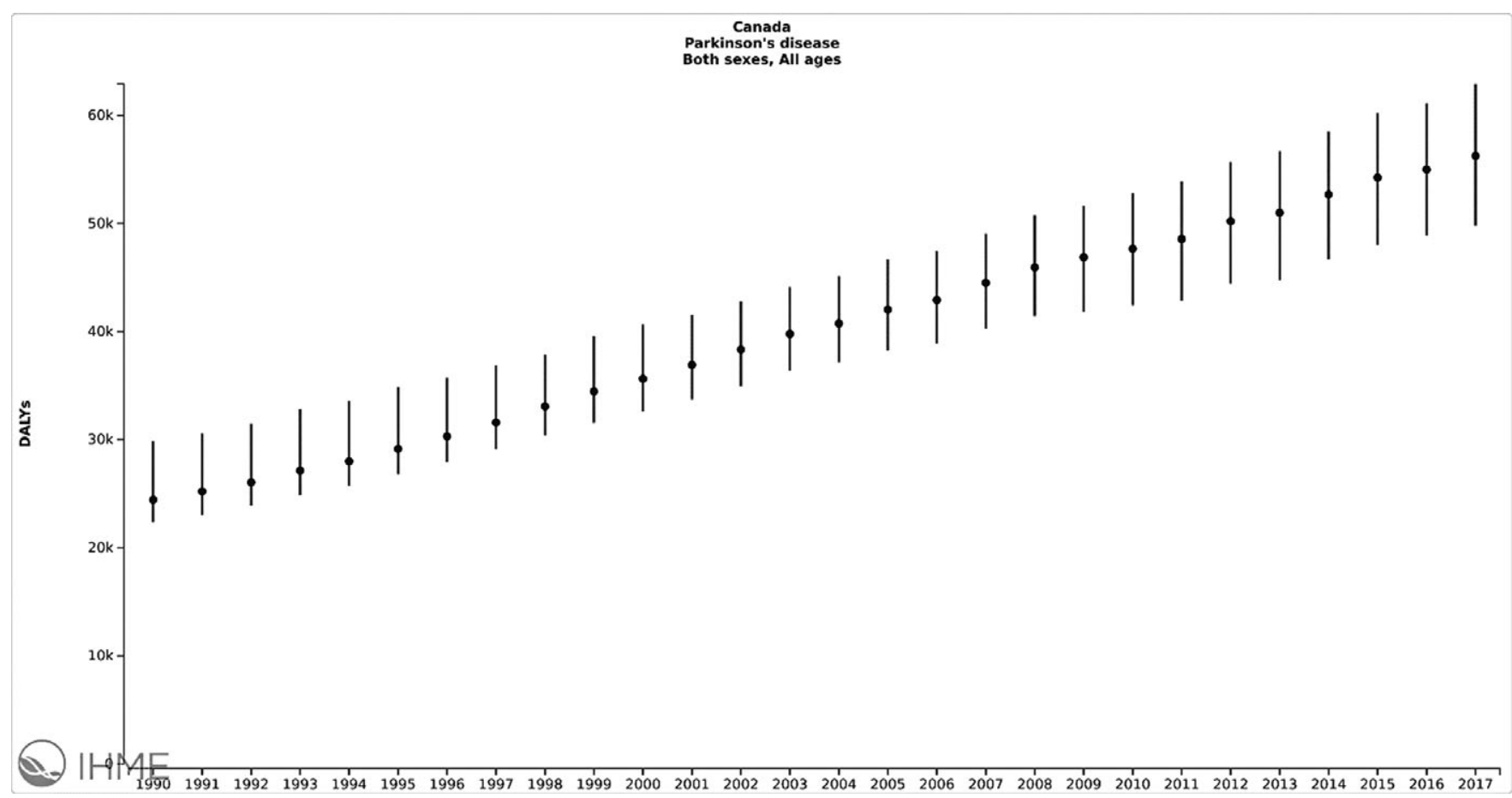

Figure 1: Doubling of the percentage of PD associated disability-adjusted life years (DALYs) in Canada from 1990-2017; (Source, GBD data; Institute for Health Metrics and Evaluation (IHME) public sources under the Creative Commons Attribution-Noncommercial 4.0 International License). In 1990, the rate of DALYs per 100,000 population was 89.72 (Uncertainty Interval (UI) 82.11-109.51). In 2017, the rate of DALYs per 100,000 population was 156.37 (UI 138.39-174.87).

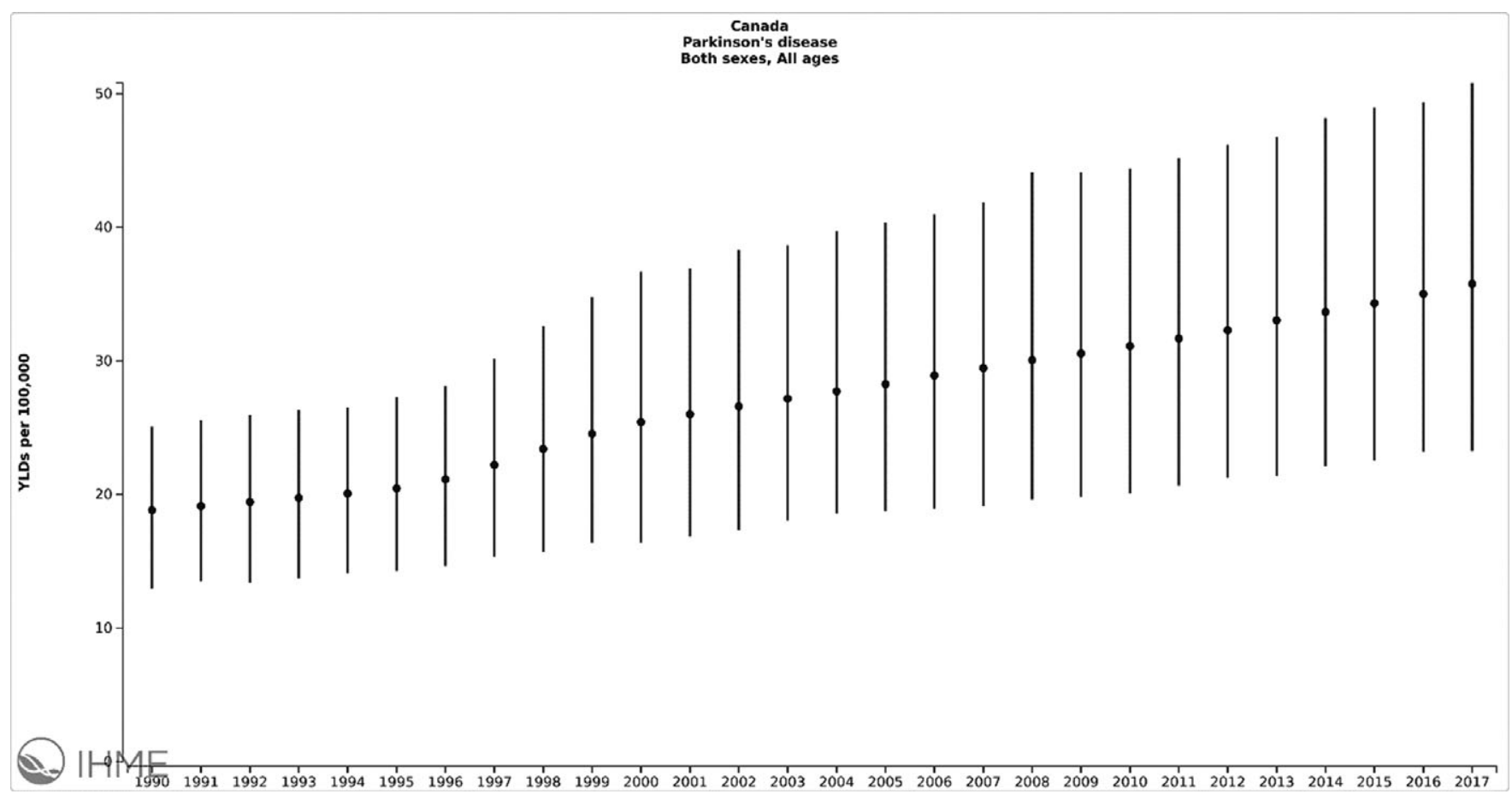

Figure 2: Doubling of PD associated years lived with disability (YLDs) in Canada from 1990-2017; (Source, GBD data; Institute for Health Metrics and Evaluation (IHME) public sources under the Creative Commons Attribution-Noncommercial 4.0 International License). In 1990, the rate of YLDs per 100,000 population was 18.83 (Uncertainty Interval (UI) 12.97-25.06). In 2017, the rate of YLDs per 100,000 population was 35.77 (UI 23.2550.82). 
in relation to the current pandemic, the need for increased funding for research in PD must be addressed globally.

None of these can be achieved without a coordinated, collaborative, advocacy action plan and execution. WBD 2020 is an ideal opportunity to act immediately.

Our primary focus is to advocate five key messages for PD:

\section{Key Messages}

Prevalence: PD is a chronic, neurodegenerative brain disease that affects more than seven million people of all ages worldwide, and its prevalence continues to increase.

Disability: PD is a whole-body disease that affects the mind, movement, and almost all aspects of brain function, with symptoms worsening over time.

Standard of care: Access to quality neurological care, lifechanging treatments, and essential medication is unavailable in many parts of the world.

Research: Additional resources are needed to help unlock the cause, onset, progression, and treatment of this disease across all ages.

Advocacy: Let us work together to diagnose PD earlier, treat it more effectively, and improve the lives of both those living with PD and their caregivers.

This WBD, the WFN seeks to improve awareness of PD. Material such as a PowerPoint slide kits, web banners, social media posts, posters, and promotional videos can be downloaded by anyone with an interest in advocacy. In the last few years, the WFN has partnered with other international organisations in campaigning. This year, we have partnered with the International Parkinson and Movement Disorder Society (MDS). We would also like to invite the Canadian Neurological Sciences Federation and your readers to consider playing a part in this year's WBD campaign: moves to end PD. You can participate by doing something as small as posting our banner ad and sharing our messages and videos (https://wfneurology.org/wbd-2020-videos?
videoId=124\&slideNum=2), or you can partner with local neurology societies and organisations for further advocacy. The websites of the WFN and MDS have educational resources freely available for anyone, so that you too can conduct a successful WBD campaign.

Advocacy is a key part of our jobs. ${ }^{4,5}$ We encourage all readers to join us with our ambitious campaign to end PD. Let us bring the best human qualities out of this global pandemic and work together to build a better world with better brain health.

\section{Disclosures}

The authors have no conflicts of interest to declare.

\section{Author COnTribution}

TW and SF conceived and presented the idea. TW drafted the initial manuscript. SF edited and contributed to the final manuscript. Both TW and SF approved the final manuscript that was submitted.

\section{REFERENCES}

1. Collaborators GBD. Global, regional, and national burden of neurological disorders, 1990-2016: a systematic analysis for the Global Burden of Disease Study 2016. Lancet Neurol. 2019;18(5): 459-80.

2. Dorsey ER, Elbaz A, Nichols E, Abd-Allah F, Abdelalim A, Adsuar $\mathrm{JC}$, et al. Global, regional, and national burden of Parkinson's disease, 1990-2016: a systematic analysis for the Global Burden of Disease Study 2016. Lancet Neurol. 2018;17(11): 939-53.

3. Wong SL, Gilmour H, Ramage-Morin PL. Parkinson's disease: Prevalence, diagnosis and impact. Health Rep. 2014;25(11): $10-4$.

4. Wijeratne T, DePold Hohler A. Residency training: advocacy training in neurology: lessons from the Palatucci Advocacy Leadership Forum. Neurology. 2013;80(1):e1-3.

5. Grisold W, Struhal W, Grisold T. Advocacy in neurology. Oxford: Oxford University Press; 2019. 